This is the accepted manuscript oh the article which has been published in final format at https://doi.org/10.1016/j.biosystemseng.2017.12.007

(C)2018.

This manuscript version is made available under the CC-BY-NC-ND 4.0 license http://creativecommons.org/licenses/by-nc-nd/4.0/ 


\title{
A cluster-graph model for herd characterisation in dairy farms equipped with
} Automatic Milking System

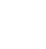

\author{
Filippo Bonora, Stefano Benni*, Alberto Barbaresi, Patrizia Tassinari, Daniele Torreggiani
}

Department of Agricultural Sciences, University of Bologna - V.le Fanin 48, 40127 Bologna, Italy

${ }^{*}$ Corresponding author: Stefano Benni,
Department of Agricultural Sciences, University of Bologna - V.le Fanin 48, 40127 Bologna, Italy, phone+390512096166, email stefano.benni@unibo.it

\begin{abstract}
The analysis of data recorded by Automatic Milking System (AMS) in dairy livestock barns has a great potential for herd management and farm building design. A big amount of data about milk production and cow welfare is available from milking robot and many researches are focusing on them in order to find relationships and correlations among the various parameters.
\end{abstract}

The goal of the study is to develop and test an innovative procedure for the comprehensive analysis of AMS-generated multi-variable time-series, with a focus on herd segmentation, aiming to support dairy livestock farm management. In particular, the study purpose is to develop and test a clustergraph model using AMS-generated data, designed to provide an automatic grouping of the cows based on production and behavioural features. First, a $k$-means cluster analysis has been implemented to the average of the time series of the main parameters recorded for each cow by AMS in a barn in Italy over a summer period. Then, all the resulting subgroups have been converted in a network and a cluster-graph analysis has been applied in order to find herd-descriptive subgraphs.

The results of the study have the potential impact of improving herd characterisation and lending support to cow monitoring and management. Furthermore, this method could represent a feasible procedure to convert alphanumeric data in a simple graphic visualization of the herd without losing the quantitative information about every single animal. 


\section{Nomenclature}

\begin{tabular}{|c|c|}
\hline Symbol & Description \\
\hline$\alpha$ & Activity \\
\hline$\alpha_{h}$ & Average hourly activity rate \\
\hline$c_{i, j}$ & Centroid of cluster $j$ for the parameter $i$ \\
\hline$C_{i, j}(\cdot)$ & Calue of cluster $j$ for the parameter $i$ \\
\hline$C i n$ & Cow Bodentification number \\
\hline$C b$ & Average daily activity \\
\hline$d \alpha$ & Milking regularity, h \\
\hline$L_{h}$ & Milk yield, l \\
\hline$M r$ & Parity \\
\hline$M y$ & Date and time of the cow passage \\
\hline$P a$ & Number of daily milking \\
\hline$t c p$ & Similarity index \\
\hline$\# M$ & Weight of the link between two nodes \\
\hline$S_{i}$ & Automatic Milking System \\
\hline$W$ & Precision Livestock Farming \\
\hline Abbreviation &
\end{tabular}

Keywords: Precision Livestock Farming; productivity; dairy cow; cluster

Information and communications technology (ICT) has become increasingly more popular in agriculture and its application in Precision Livestock Farming (PLF) has increased rapidly in the last decades, especially in dairy cow barns. It is broadly acknowledged that the main expected benefits from PLF deal with real-time monitoring of animal welfare and health, early disease alerting, increase in milk yield, reduction in production costs, and improvement of farmers' work conditions and quality of life (Berckmans, 2014). As it is well known, the introduction of Automatic Milking Systems (AMS) in the late 1990s has deeply changed the barn layout and the 
herd management in dairy farms (Rodenburg, 2017), and it has been considered one of the earliest precision livestock farming developments (John et al., 2016). In the last years, many researches have focused on data from AMS-equipped livestock farms, with the aim of improving and optimizing the different aspects of the farmers' work. For example, Gaworski, Leola, Sada, Kic, \& Priekulis, (2016) studied the relationships between cow traffic systems and the efficiency of the AMS use, while Kaihilahti, Suokannas, and Raussi (2007) associated a video recording technology with the automatic milking system to observe the deviations in the milking process. Other researchers have investigated the performances of the milking robot according to different feed deliveries (six times per day compared to twice daily, both via automatic feeding system, AFS) (Oberschätzl-Kopp, Haidn, Peis, Reiter, Bernhardt, 2016) and the variation in lying times of the cows in AMS farms (Westin et al., 2016). Moreover, the introduction of AMS has provided new knowledge about the proliferation and the detection of some cow diseases, among which mastitis diagnosis deserves particular attention(Castro, Pereira, Amiama, Bueno, 2015; Lehmann, Wall, Wellnitz, Bruckmaier, 2015; Steeneveld, Vernooij, Hogeveen, 2015).

AMSs measure specific data about milk production and cow behaviour, providing farmers with useful real-time information for each cow. The remarkable number of records stored in an AMS database has a great potential for herd characterisation and management optimisation is still largely underexploited. In particular, data from AMS are suitable to identify clusters within the herd, based on the most informative variables in terms of cow productivity and welfare. In this regard, dairy cattle cluster analysis has only recently been investigated, specifically to assess physical activity of cows while taking into account environmental conditions (Adamczyk, Cywicka, Herbut, Trześniowska, 2017). This approach is considered as a technical support to cattle breeders at the stage of data collection and analysis, and for the assessment of rearing conditions. In this context, the development of cluster-graph models for a detailed herd characterisation and cow classification 
based on AMS-generated time series of data appears to be a significant contribution, and it represents an approach which has yet to be found in the scientific literature.

Within these challenging research topics, the goal of this study is to develop and test innovative procedures for the comprehensive analysis of AMS-generated multi-variable time-series with a focus on herd segmentation in order to support livestock farm management. In particular, the specific aim is to develop and test a cluster-graph model using AMS-generated data, designed to provide an automatic grouping of the cows based on production and behavioural features.

\subsection{The Study Case}

\section{MATERIALS AND METHODS}

A dairy farm equipped with AMS was adopted for the development of the cow clustering method. This farm is placed in the municipality of Budrio, about $15 \mathrm{~km}$ north-east of Bologna (Emilia-

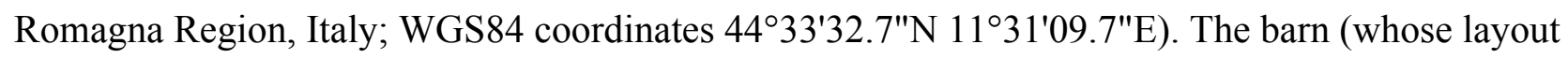
is shown in Fig, 1) was $51 \mathrm{~m}$ long and $23 \mathrm{~m}$ wide rectangular building, with SW-NE-oriented longitudinal axis, consisting of a hay storage area on the SE side, a resting area in the central part of the building, and a feeding area and a feed delivery lane on the NW side. The resting area, whose floor was partially slatted, hosts 78 cubicles with straw bedding where about 65 Friesian cows were housed; two blocks of head-to-head cubicles located in its central part and another row that ran along the entire length of the resting area. The milk-room was located on the SW side of the building, next to the offices and technical plant rooms. Ventilation was controlled by three highvolume low-speed (HVLS) fans with five horizontal blades activated by a temperature-humidity sensor situated in the middle of the barn. Cow milking was performed by means of a robotic milking system (marked in the right part of Fig, 1) "Astronaut A3 Next" by Lely, Maassluis, The Netherlands. The robot was programmed to assure a number of daily visits for each cow depending 
on the cow productivity and its expected optimal milk yield per visit, with a minimum and a maximum number of daily visits as constraints.

\subsection{Data Acquisition and Preliminary Data Analysis}

Cow-related and milk production data of farm A have been recorded by the AMS at each cow passage. Based on the database downloaded from the AMS management software, a matrix designated 'Visit' was created for each cow where each row corresponds to a cow passage and the columns contain the following parameters: Cow Identification Number (Cin), Date and Time of the Cow Passage (tcp), Milk Yield (My), Cow Body Weight $(\mathrm{Cb})$ and Parity $(\mathrm{Pa})$.

Cows behaviour data was measured by means of a collar by SCR (Netanya, Israel) mounted for the cow identification. It monitored activity levels $(\alpha)$ of each animal by means of an acceleration sensor measuring the duration and the intensity of the movements. This parameter was recorded in 2 hour blocks and it is the parameter usually used in livestock management for automated heat detection (Shahriar et al., 2016). Data were downloaded from the collars at each passage through the AMS robot and collected in a matrix designated 'Activity', where each row contained the twohour activity of each cow.

'Visit' and 'Activity' matrices were jointly processed. Since milking with AMS is voluntary, milking events, data acquisition frequency and temporal distribution are highly variable, so that the datasets cannot be compared directly on a regular basis. To allow a comparison of synchronous data of the parameters recorded by AMS and thus to perform preliminary analyses, milk production and activity data were interpolated over synchronous 6-hour time steps (12 am - 6 am, 6 am - 12 pm, 12 pm - 6 pm, 6 pm - 12 am). This sampling has been selected in order to mostly avoid repetitions of data from the same animal in the same time step. The average hourly rate of milk production $\left(L_{h}\right)$ and activity $\left(\alpha_{h}\right)$ for each cow have been calculated according to the following Eq. 1 and 2: 


$$
L_{h}\left(t c p_{\mathrm{i}}\right)=\frac{M y_{i}-M y_{i-1}}{t c p_{i}-t c p_{i-1}}
$$

$$
\alpha_{h}\left(t c p_{\mathrm{j}}\right)=\frac{\alpha_{j}-\alpha_{j-1}}{t c p_{j}-t c p_{j-1}}
$$

113 where $t c p_{i}$ represent the date and time of the $i$ th cow passage.

114 Then, interpolating $L_{h}$ and $\alpha_{h}$ over $6 \mathrm{~h}$ time spans, a continuous trend line of milk production and activity for each cow were obtained. Before interpolation and data aggregation, rows with null milk yield or feed intake data were deleted, since they corresponded to unsuccessful milking sessions and they would have led to biased values (e.g. when a stressed or uncomfortable cow kicks the machine). Similarly, milking events closer than $4 \mathrm{~h}$ for the same cow were aggregated to obtain a smoother distribution of data.

Gaussian normality of milk production and activity data for each cow was tested both graphically and by means of the Jarque-Bera test (Jarque \& Bera, 1980) for fixed time steps, i.e. the normality of $L_{h}$ and $\alpha_{h}$ has been checked for each cow. It proved that their distributions could be considered as normal, with a significance level of rejection of null hypothesis fixed at $5 \%$. Therefore, the means and the standard deviations were considered significant to characterise in a synthetic form the milk production and activity data of the herd and their distributions.

\subsection{Clustering Method}

Cow clustering based on production and behavioural features has been performed focusing on data surveyed in summer 2015 (from June $21^{\text {st }}$ to September $30^{\text {th }}$ ). It is worth noticing that the total number of analysed cows (88) accounts for all the animals reared in the barn in the considered study period. The $k$-means algorithm (MacQueen, 1967) has been used to study the following parameters in the study period: 
- Number of daily milking (\#M);

- Parity $(P a)$;

- Average daily activity $(d \alpha)$

- Milking regularity, in terms of standard deviation of the time intervals between milking events in the study period $(M r)$;

- Cow body weight $(C b)$.

In particular, each cow was represented by the mean values for each parameter in the above five $k$ means analyses. This approach allowed the characteristics of the animals to be described in a stationary and concise manner during the study period. For each variable, different $k$ values were selected "a posteriori" to highlight some particular trends.

The clusters obtained through the five $k$-means algorithms were joined in a network graph with Gephi (0.9.1 version), an open source and cross-platform exploration tool for networks and complex systems (Bastian, Heymann, Jacomy, 2009). The network was designed by assigning each cow a node, and linking two nodes if the cows belong to the same cluster (referring at least to one clustering variable). The weight of the link, $W$, between two nodes $A$ and $B$ was defined by the summation of the five "similarity index" $S_{i}$ as follows:

$$
W(A, B)=\sum_{i=1}^{5} S_{i}(A, B)
$$

where $i$ identified one of the previous five variables, $A$ and B two were general cows and $S_{i}$ was calculated for each parameter $i$ as

$$
S_{i}(A, B)=\left\{\begin{array}{rr}
1-\frac{\left|C_{i, j}(A)-C_{i, j}(B)\right|}{c_{i, j},} & \text { if } j=j^{\prime} \\
0, & \text { if } j \neq j^{\prime}
\end{array}\right.
$$


The resulting network was analysed and processed to find subnetworks based on modularity, i.e. a measure which minimises the number of edges from two different clusters (Newman, 2006): this procedure produced the final graph.

The methodology was thus composed of three steps:

1. Development of five $k$-means analysis, one for each parameter described above;

2. Creation of a network with nodes and a measure of connectivity;

3. Analysis of the network in order to find subnetworks.

This differs from a classic $k$-means cluster methodology and allows:

- The distribution of every single parameter within the herd to be analysed, choosing for each one the most appropriate $k$;

- A network to be designed to convert numbers and matrices into nodes and colours, i.e. a more immediate and easy way for the farmer to monitor the herd and the distribution of animals into clusters. Moreover, the clustering approach based on modularity does not require to define "a priori" the number of clusters the dataset has to be subdivided into.

\section{RESULTS AND DISCUSSION}

The results of herd clustering of farm A according to the single descriptive variables are reported in Tables 2, 3, 4, 5, and 6 . In Table 2, the cluster analysis provided four groups of cows with different milking habits in terms of mean daily events. In Table 3, the herd was subdivided into six groups depending on parity. The group composed by first-calf cows $\left(C 1_{P a}\right)$ and that of cows with parity equal to $2\left(C 2_{P a}\right)$ have similar cardinality and together represent the largest part of the herd. Table 4 shows that the herd could be divided into four groups of animals with similar cardinality and 
175 significantly different levels of activity. Table 5 points out the different habits of herd for the act of

176 milking, in terms of standard deviation of time intervals between two milking events in succession.

177 Finally, Table 6 spotlights the differences within the herd in terms of body mass.

178 The final modularity process led to the identification of three clusters which are shown in Fig. 2,

179 where the graph is drawn based on the "Force Atlas", the Noack's edge-directed force layout (Gephi

180 Consortium, 2011), adopting the following values of the ruling parameters: Repulsion equal to

181 10,000; Gravity was equal to 400 and adjusted by size. The size of each node was proportional to

182 daily milk yield: the highest was the milk yield, the longer was the node radius. Parity is indicated

183 as a number inside each circle.

184

Table 2. Statistics about clusters of mean number of daily milking.

\begin{tabular}{cccccc}
\hline Cluster & Cardinality & Min & Max & Median & Centroid \\
\hline$C 1_{\# M}$ & 10 & 1.2 & 1.7 & 1.5 & 1.5 \\
$C 2_{\# M}$ & 38 & 1.8 & 2.3 & 2.0 & 2.0 \\
$C 3_{\# M}$ & 30 & 2.3 & 2.8 & 2.6 & 2.6 \\
$C 4_{\# M}$ & 10 & 2.9 & 3.7 & 3.0 & 3.1 \\
\hline
\end{tabular}


Table 3. Statistics about clusters of number of parity.

\begin{tabular}{ccc}
\hline Cluster & Cardinality & Number of parity \\
\hline$C 1_{P a}$ & 35 & 1 \\
$C 2_{P a}$ & 33 & 2 \\
$C 3_{P a}$ & 10 & 3 \\
$C 4_{P a}$ & 7 & 4 \\
$C 5_{P a}$ & 2 & 5 \\
$C 6$ & 1 & 6 \\
\hline
\end{tabular}

Table 4. Statistics about clusters of mean daily activity of cows.

\begin{tabular}{cccccc}
\hline Cluster & Cardinality & Min & Max & Median & Centroid \\
\hline$C 1_{\alpha}$ & 21 & 28.4 & 44.5 & 40.7 & 39.6 \\
$C 2_{\alpha}$ & 24 & 45.8 & 54.9 & 49.7 & 50.2 \\
$C 3_{\alpha}$ & 19 & 55.3 & 65.9 & 59.3 & 60.0 \\
$C 4_{\alpha}$ & 14 & 66.8 & 83.8 & 72.1 & 72.2 \\
\hline
\end{tabular}




\begin{tabular}{cccccc}
\hline Cluster & Cardinality & Min (h) & Max (h) & Median (h) & Centroid (h) \\
\hline$C 1_{M r}$ & 4 & 0 & 1.3 & 0.7 & 0.7 \\
$C 2_{M r}$ & 39 & 1.7 & 3.0 & 2.5 & 2.5 \\
$C 3_{M r}$ & 26 & 3.2 & 4.4 & 3.7 & 3.7 \\
$C 4_{M r}$ & 14 & 4.5 & 6.3 & 5.2 & 5.2 \\
$C 5_{M r}$ & 5 & 7.0 & 9.6 & 7.3 & 7.7 \\
\hline
\end{tabular}

Table 5. Statistics about clusters of standard deviation of time intervals (in hour) between milking events in succession.
200

201 202 
expectations. The remaining two groups had similar mean parity, between two and three, and mostly differ as for number of milking events and milking regularity.

More specifically, cluster 1 has a small number of effective AMS visits (daily average of 2) with poor regularity (standard deviation of time interval between visits of almost $5 \mathrm{~h}$ ). This cluster included nearly half of the animal population. By contrast, cluster 3 has a significantly smaller cardinality than the other two groups, but it was strongly characterised by good milking performances. These are expressed by the average number of daily AMS visits which exceeded three and by their good regularity, given by a standard deviation of the time intervals was about one half of that of cluster 1 . It is interesting to observe that the mean activity score of this cluster was significantly higher than that of the other ones, while the average body mass was lower than cluster 1.

An important confirmation of the diversification of the three clusters in terms of productive characteristics is provided in Tables 7 and 8, which contain the synthetic indexes of the main data regarding cow productivity, including the averages and standard deviations of daily milk yields of the clusters, besides their cardinality. Milk yield was not selected as a parameter for clustering because it was considered as the dependent variable according to which the effectiveness of the clustering procedure and the productive feature of each resulting group of cows was assessed. The results in terms of number of daily milking events, daily milk yields and milking regularity neatly characterised the three clusters according to three different levels of productivity. Therefore, not only the clustering method identified groups of animals with different behaviour and physical conditions, but it also provided clusters with clearly different average productivity. 
Table 7. Statistics for the final clusters (part 1): mean $(m)$ and standard deviation $(s t d)$ of each variable.

\begin{tabular}{|c|c|c|c|c|c|c|}
\hline \multirow[b]{3}{*}{ Cluster } & \multicolumn{2}{|c|}{$\# M$} & \multicolumn{2}{|c|}{$\mathrm{Pa}$} & \multicolumn{2}{|c|}{$d \alpha$} \\
\hline & \multicolumn{2}{|c|}{ \# daily milking } & \multicolumn{2}{|c|}{ number of parity } & \multicolumn{2}{|c|}{ average daily activity } \\
\hline & $m$ & std & $m$ & std & $m$ & std \\
\hline 1 & 2.0 & 0.4 & 2.7 & 1.0 & 53.5 & 9.8 \\
\hline 2 & 2.3 & 0.3 & 1.0 & 0.0 & 53.9 & 12.9 \\
\hline 3 & 3.0 & 0.3 & 2.4 & 0.7 & 60.2 & 13.0 \\
\hline
\end{tabular}

Table 8. Statistics for the final clusters (part 2): mean $(\mathrm{m})$ and standard deviation $(\mathrm{std})$ of each variable.

\begin{tabular}{cccccccc}
\hline & \multicolumn{2}{c}{$M y$ (litre) } & \multicolumn{2}{c}{$M r$ (hour) } & \multicolumn{2}{c}{$C b(\mathrm{~kg})$} & \\
& \multicolumn{2}{c}{ daily milk yield } & milking regularity & \multicolumn{2}{c}{ cow mass } & \\
\cline { 2 - 6 } Cluster & $m$ & std & $m$ & std & $m$ & std & Cardinality \\
\hline 1 & 24.7 & 10.0 & 4.3 & 1.5 & 674.5 & 58.0 & 43 \\
2 & 27.0 & 10.4 & 2.9 & 1.3 & 587.4 & 33.0 & 35 \\
3 & 36.7 & 7.0 & 2.2 & 0.3 & 646.4 & 66.8 & 10 \\
\hline
\end{tabular}

The average milk yield of the whole herd was $271 \mathrm{~d}^{-1}$ and can be considered as a reference value to 239 assess the results of each cluster. In fact, cluster 3 produced a higher milk yield than clusters 1 and 2. The performance of cluster 2 was almost $101 \mathrm{~d}^{-1}$ below cluster 3 and represented the intermediate 241 result. This negative difference was likely to be due to the condition of first delivery of the cows.

242 The worst average performance was given by cluster 1, which mostly included animals with scarce 243 productivity. 
The results highlight that lactating cows have very diversified conditions and that cluster analysis is an effective tool to identify the most significant groups if data about animal behaviour and conditions are available, which is in the case of AMS use. The identification of clusters can contribute to defining feeding strategies based not only on the milk yield and lactation period, but also on the other descriptive variables, which prove to be effective in characterising herd groups with different production potentials. The results suggest also that particular attention should be paid to single animals whose milk yield is poorly consistent with the average value of the cluster they belong to. In particular, in cluster 1 those cows that showed high milking values should be monitored in order to prevent their production decreasing and reaching the common values of that cluster. In this case, proper measures should be defined to increase the number of daily visits to AMS and their regularity in time. In cluster 3, those cows which showed milking production significantly below the cluster mean represent another aspect deserving attention: proper investigations should be carried out to identify the causes of low milk yield and to identify possible corrective strategies. Finally, in cluster 2 those cows with the lowest production rates should be checked to verify if this is due to their normal lactating curve pattern or if there are other factors hampering their expected productivity. The resulting clusters may be farm-dependent, but the methodology developed here, consisting of a computational procedure and an approach to a critical analysis of the results, has general validity and it represents a supplemental tool for providing highly informative real time knowledge of the herd condition.

\section{CONCLUSIONS}

A numerical approach to data analysis of dairy farms has been developed through the formulation of a proper integrated model to process production and behavioural data of every cow. A clustergraph analysis method has been applied to a dataset derived from AMS source, containing the time series of the main parameters recorded for each cow in the summertime in a barn in Italy. The 
combination of cluster analysis and graph theory has allowed objective results to be obtained using statistical and numerical methodologies: the herd studied was characterised according to three main clusters, which proved diverse from each other in terms of productivity and animal behaviour. The specific results of the cluster analysis over the study case obviously depends on the herd characteristics, but the methodology were developed according to general criteria that are independent of the single case of application. Therefore, the method appears to be directly useful for farmers under various operational conditions.

The results lend support to cow monitoring, through the comparison between the measured and expected cow behaviour. Herd partitioning could help to regulate the number of milking events or the supplementary feeding of specific groups, and the identification of the clusters can contribute to define proper feeding strategies. These should be based not only on the milk yield and lactation period, as it usually occurs, but also on the other descriptive variables recorded by the AMS, which proved to be effective in characterising the herd groups. Moreover, the proposed cluster analysis is also a method to indicate a correlation between the behaviour patterns characterised by the variables adopted for clustering and milk yields. This issue is worth of further investigation through the development of innovative numerical models.

As for the aspects related to the evolution in time of the herd composition and the characteristics of the cows, the method proposed considers a classification of a cow to a group which is constant over the time period analysed. Nevertheless, the method is suitable to investigate the clustering of cows also with reference to a number of consecutive shorter periods and thus to analyse and characterise the evolution of the composition of the clusters in a dynamic way and to identify animals with anomalous trends.

Further developments of the research are ongoing and firstly consist in the application of the model to other farms in different geographic contexts and under different climatic and farming conditions for a finer calibration. Moreover, the integration of these results with video or RFID position 
294 analysis could provide a better comprehensive behavioural description of dairy cattle in a farm.

295 Finally, the developments of the research are focusing also on the implications of cows clustering

296 for innovations in the definition of spatial layouts, with expected benefits for the design of dairy

297 barns.

\section{ACKNOWLEDGMENTS}

299 The study has been developed within research agreements between the Department of Agricultural

300 Sciences of the University of Bologna and the dairy farm Piazzi (Budrio, BO, Italy). 
304 Fig. 1. Plan layout of the barn adopted as study case.

305 Fig. 2 Clusters of the study farm with Gephi. The graph is composed by 88 nodes and 5920 edges.

306 Numbers inside each circle represent the parity of each cow.

307 Fig. 3. Mean values of each variable for each cluster: average daily milking actions, number of 308 parity; (average daily activity)/10; standard deviation of time lag in hour between milking; mass in 309 hundreds of $\mathrm{kg}$. 
Adamczyk, K., Cywicka, D., Herbut, P., \& Trześniowska, E. (2017). The application of cluster analysis methods in assessment of daily physical activity of dairy cows milked in the Voluntary Milking System. Computers and Electronics in Agriculture, 141, 65-72. http://doi.org/10.1016/j.compag.2017.07.007

Bastian, M., Heymann, S., \& Jacomy, M. (2009). Gephi: An Open Source Software for Exploring and Manipulating Networks. Third International AAAI Conference on Weblogs and Social Media, 361-362. http://doi.org/10.1136/qshc.2004.010033

Berckmans, D. (2014). Precision livestock farming technologies for welfare management in intensive livestock systems. Rev. Sci. Tech. Off. Int. Epiz, 33(1), 189-196. http://doi.org/10.20506/rst.33.1.2273

Castro, A., Pereira, J. M., Amiama, C., \& Bueno, J. (2015). Mastitis diagnosis in ten Galician dairy herds ( NW Spain ) with automatic milking systems, 13(4).

Gaworski, M., Leola, A., Sada, O., Kic, P., \& Priekulis, J. (2016). Effect of cow traffic system and herd size on cow performance and automatic milking systems capacity. Agronomy Research, $14(1), 33-40$.

Gephi Consortium. (2011). Gephi Tutorial Layouts. Gephi Consortium.

Jarque, C. M., \& Bera, A. K. (1980). Efficient tests for normality, homoscedasticity and serial independence of regression residuals. Economics Letters, 6(3), 255-259. http://doi.org/10.1016/0165-1765(80)90024-5

John, A. J., Clark, C. E. F., Freeman, M. J., Kerrisk, K. L., Garcia, S. C., \& Halachmi, I. (2016). Review: Milking robot utilization, a successful precision livestock farming evolution. Animal, 10(9), 1484-1492. http://doi.org/10.1017/S1751731116000495

Kaihilahti, J., Suokannas, A., \& Raussi, S. (2007). Observation of Cow Behaviour in an Automatic 
Milking System using Web-based Video Recording Technology. Biosystems Engineering, 96(1), 91-97. http://doi.org/10.1016/j.biosystemseng.2006.10.001

337

Lehmann, M., Wall, S. K., Wellnitz, O., \& Bruckmaier, R. M. (2015). Changes in milk L-lactate, lactate dehydrogenase, serum albumin, and IgG during milk ejection and their association with somatic cell count. Journal of Dairy Research, 82(2), 129-134. http://doi.org/10.1017/S002202991400065X

MacQueen, J. B. (1967). Kmeans Some Methods for classification and Analysis of Multivariate Observations. 5th Berkeley Symposium on Mathematical Statistics and Probability, 1(233), 281-297. http://doi.org/citeulike-article-id:6083430

Newman, M. (2006). Modularity and community structure in networks. Proceedings of the National Academy of Sciences, 103(23), 8577-82. http://doi.org/10.1073/pnas.0601602103

Oberschätzl-Kopp, R., Haidn, B., Peis, R., Reiter, K., \& Bernhardt, H. (2016). Studies on dairy cow behaviour with automatic feeding in a herd milked by an AMS. Landtechnik, 71(2). http://doi.org/10.15150/lt.2016.3122

Rodenburg, J. (2017). Robotic milking: Technology, farm design, and effects on work flow. Journal of Dairy Science, 100(9), 7729-7738. http://doi.org/10.3168/jds.2016-11715

Shahriar, M. S., Smith, D., Rahman, A., Freeman, M., Hills, J., Rawnsley, R., Henry, D., BishopHurley, G. (2016). Detecting heat events in dairy cows using accelerometers and unsupervised learning. Computers and Electronics in Agriculture, 128, 20-26. http://doi.org/10.1016/j.compag.2016.08.009

Steeneveld, W., Vernooij, J. C. M., \& Hogeveen, H. (2015). Effect of sensor systems for cow management on milk production, somatic cell count, and reproduction. Journal of Dairy Science, 98(6), 3896-905. http://doi.org/10.3168/jds.2014-9101

Westin, R., Vaughan, A., de Passillé, A. M., DeVries, T. J., Pajor, E. A., Pellerin, D., Siegford, 

automatic milking systems and the relation to lameness, leg lesions, and body condition score. 


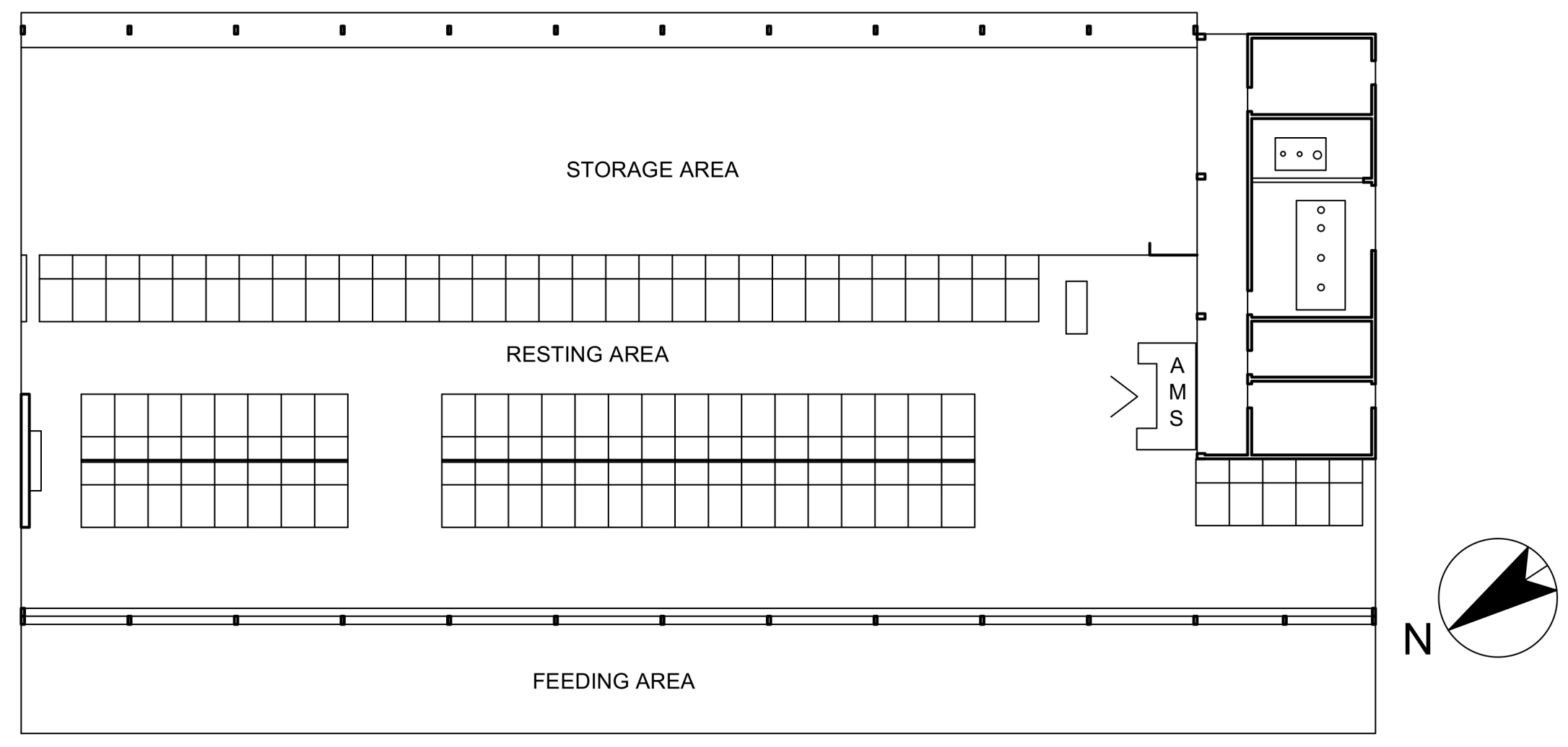




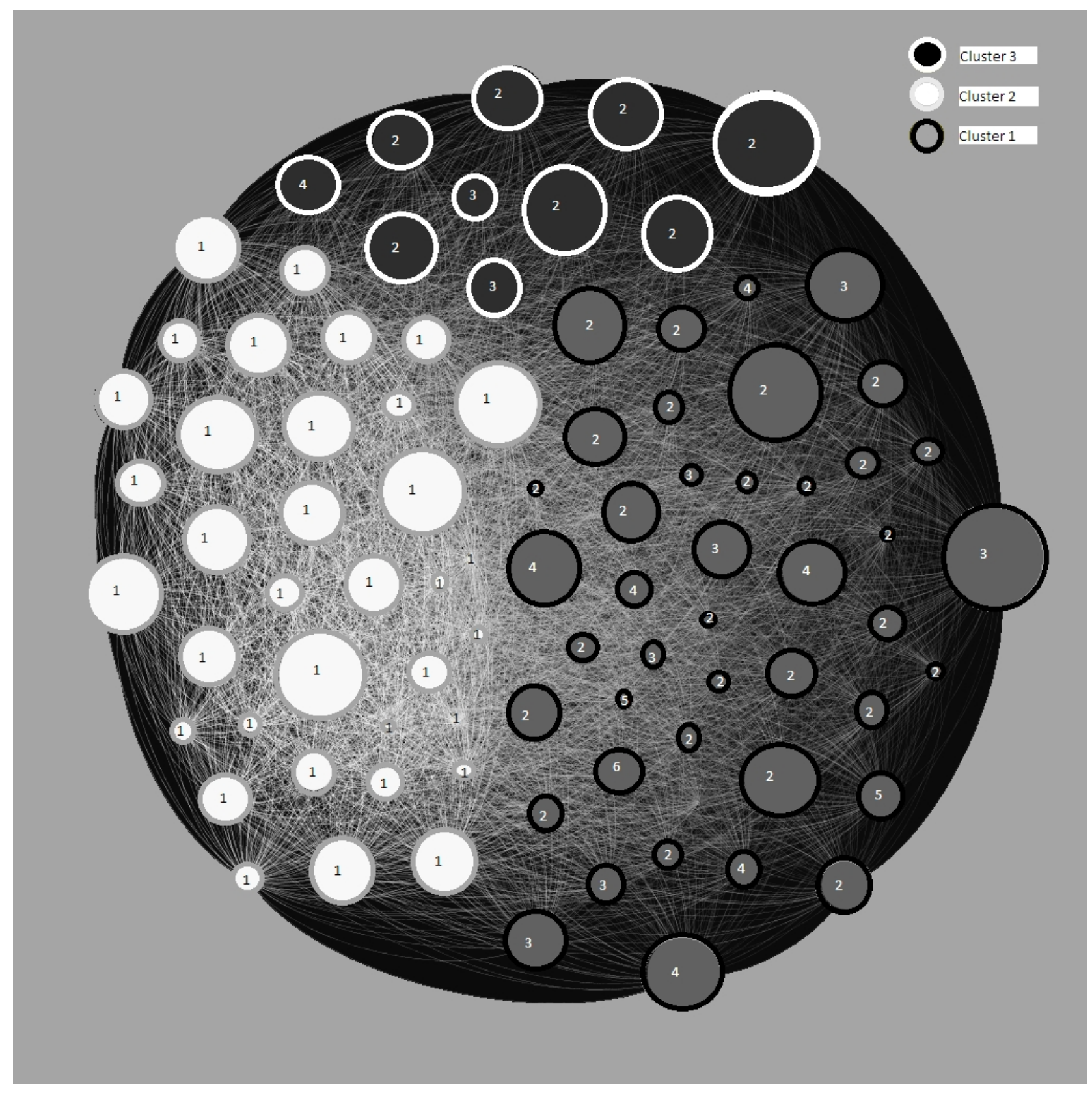




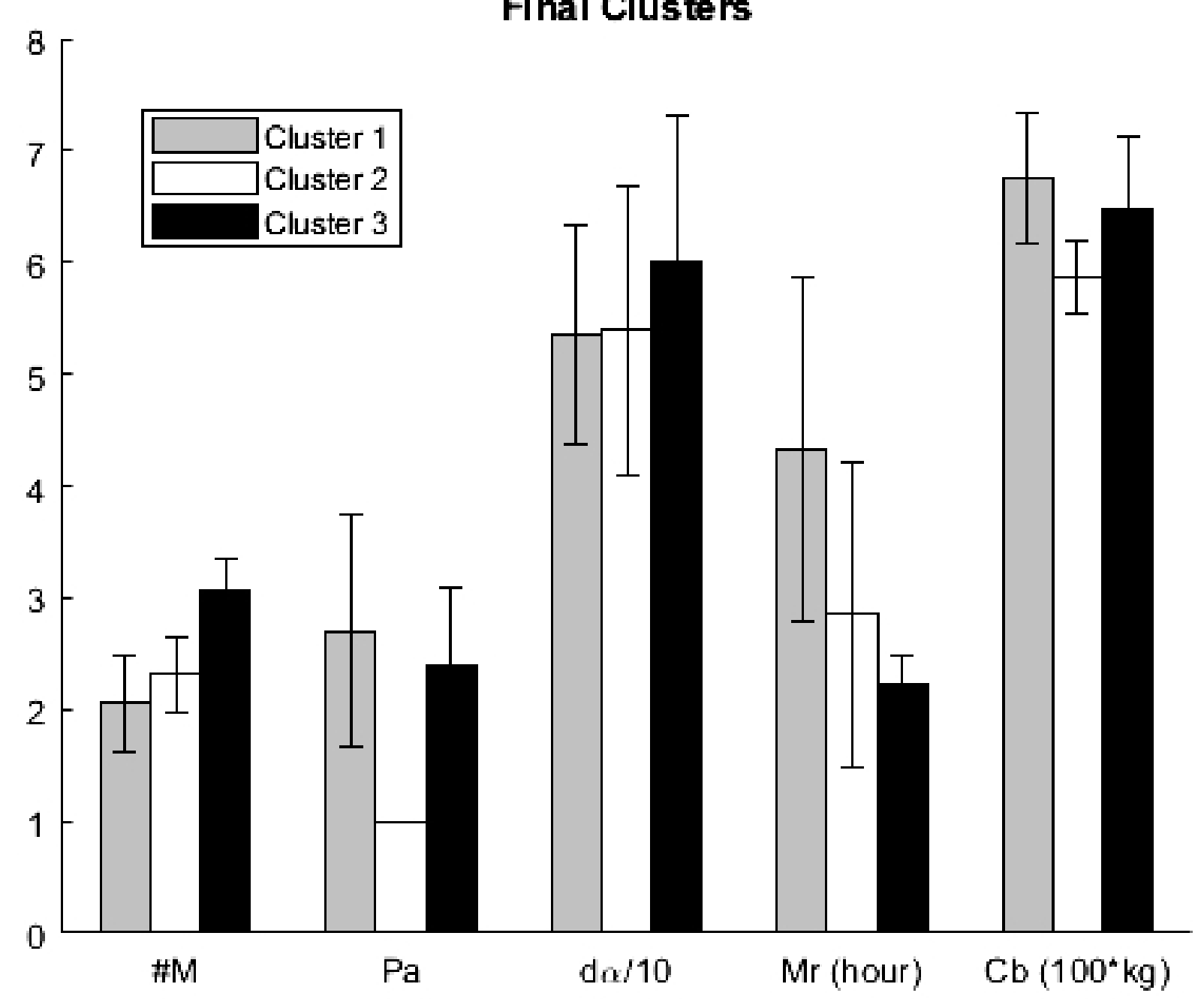

